\title{
Survey on housing, management and welfare of dairy cattle in tie-stalls in western Italian Alps
}

\author{
S. MATTIELLO, D. ARDUINO, M. V. TOSI \& C. CARENZI \\ Istituto di Zootecnica, Facoltà di Medicina Veterinaria, Università degli Studi di Milano, Milan, Italy
}

\begin{abstract}
In the Alps, the traditional breeding system for dairy cattle is based on the alternation between a free-ranging period on mountain ranges during the summer, and an indoor period in tie-stalls in the winter. Several welfare issues may arise in tiestall housing systems. We describe the situation in 47 farms in three villages in western Italy, trying to identify possible relationships among structural and management characteristics, animal health and behaviour traits. A long duration of the grazing period, associated with frequent manure removal during the housing period, are probably key factors for limiting the occurrence of lameness. Teat trauma is more common in narrower stalls. Getting-up behaviour is unnatural in most of the visited farms. Some lack in the farmers' knowledge of animal behaviour was noted. Some structural and management characteristics are strictly related to geographical constraints. However, circumstances permitting, some expedients may be achieved for improving welfare levels.
\end{abstract}

Keywords: Dairy cows, housing system, tethering, behaviour, Italian Alps.

\section{Introduction}

In the Italian Alps, the traditional breeding system for dairy cattle is based on the alternation between an indoor period in tie-stalls in the winter and a freeranging period on mountain pastures during the summer. Cattle may also benefit from an additional outdoor grazing period in spring and in early autumn, at lower altitudes, usually in proximity to the winter housing buildings. The general perception of dairy cattle in mountain areas is that of a welfarefriendly free-ranging system, and does not take into account the housing conditions during the cold season. In terms of welfare, the housing of cattle in tie-stalls presents some disadvantages compared to loose housing, such as the limited possibility of movement, limited environmental stimuli and reduced possibility of developing social behaviour (Charron, 1998; Centro Ricerche Produzioni Animali, 1999). Furthermore, some studies also suggest that cows in tie-stalls may have a higher clinical mastitis rate, a higher disease rate and a lower fertility status (Valde et al., 1997), although these variables can obviously be affected also by other factors, such as prophylaxis and cleaning routines. Another disadvantage of tie-stalls compared to loose-housing systems is a higher incidence of podal and body lesions (Bloom, 1983), especially when cows do not have access to regular outdoor exercise (Regula et al., 2004). However, other researches show a higher prevalence of lame cows in free stalls (cubicles) rather than in tie-stalls (Cook, 2004), or no effect of the housing system on the prevalence of lameness (Alban, 1995).

Owing to the increasing importance attributed by consumers to animal welfare, several attempts to measure its level on farm have recently been achieved (e.g., for dairy cattle: Main et al., 2001; Sørensen et al., 2001; Rousing, 2003; Winckler et al., 2003).

The present study aims to provide a general picture of the current situation in an Italian alpine region, highlighting the differences in structures and management which are due to contingent geographical conditions, and investigating their impact on some animal welfare traits. To this end, we carried

Correspondence: S Mattiello, Istituto di Zootecnica, Facoltà di Medicina Veterinaria, Università degli Studi di Milano, Via Celoria 10, 20133 Milan, Italy. E-mail: silvana.mattiello@unimi.it 
out a survey in three villages, where cows are commonly housed in tie-stalls during the winter. We describe the situation and try to identify possible relationships among structural and management characteristics, animal health and behaviour traits.

\section{Materials and methods}

The study was carried out in three neighbouring villages located in Valle d'Aosta, a mountain region in the western Italian Alps. We visited 16 farms in Lillianes, 23 in Pont Saint Martin (PSM) and 8 in Perloz. PSM has a smaller surface area $\left(6.88 \mathrm{Km}^{2}\right)$ and lies in a flatter area, at a lower altitude (the chief town is at $345 \mathrm{~m}$ a.s.1.) than Perloz $\left(23 \mathrm{Km}^{2}, 660 \mathrm{~m}\right.$ a.s.1.) and Lillianes (18.88 $\mathrm{Km}^{2}, 665 \mathrm{~m}$ a.s.1.). Cows were of the local breed Valdostana Pezzata Rossa.

Data were gathered in specific evaluation forms divided into two main parts: direct remarks and a questionnaire to the farmer. Direct remarks were collected during on-the-spot inspection and were based on direct observations and measurements made by the interviewer; they included general information on the housing structures (stall size and design, feed trough size and location, tethering system, wall connections) and additional information about the presence of a bull, the level of ventilation and a subjective evaluation of smell perception (see also Appendix 1 for variables codes). As no partitions between stalls were present, stall width was calculated as the distance between two adjacent tethering junctions.

Questions to the farmer dealt with general characteristics of the farm (location, year of construction, average cow weight, etc.), management practices (milking techniques, amount of litter, modality and frequency of manure removal, duration of the grazing period, etc.) and welfare indicators (health parameters, presence of abnormal behaviour). We considered as health problems the presence in the herd of more than $10 \%$ of the cows with lameness or teat trauma during the last 12 months. The abnormal behaviours considered in this interview were tongue playing, water lapping and the getting-up movement, and they were described to the farmers during the interview. Tongue playing consists of repetitive, circular movements of the tongue inside or outside the mouth for more than 1 min (Albright \& Arawe, 1997). Water lapping is described as repeated licking at the water, instead of siphoning it up (Albright \& Arawe, 1997). We considered as a behavioural problem the persistent and repetitive presence of tongue playing and water lapping in more than 5\% of the cows (Wiepkema et al., 1983). With regard to the getting-up movement, we showed a picture with the two movements (normal and abnormal; Figure 1) to the farmers, asking them which was the more common way of getting up in their herd. Although several other parameters can be used to evaluate cows' welfare (e.g., behavioural response to humans or behaviour during milking; Waiblinger et al., 2003; Hagen et al., 2004), these were not taken into account in the present investigation, as their measurement is more time-consuming, and it was impossible for the veterinary officer who carried out this survey to spend too much time for each inspection.

The answers to the questionnaire were based on the farmer's individual estimation and the farmer was always offered the possibility to ask clarifying questions and to give personal remarks.

The questionnaire was always filled by direct interviews made by a veterinary officer (who was always the same person in order to obtain homogeneous information), during an on-farm visit. In Italy, veterinary officers work for local health boards (Aziende Sanitarie Locali) and their role is to carry out regular on-the-spot checks. With regard to animal welfare on the farm, one of their main tasks is to check on holdings in order to verify compliance with legislation.
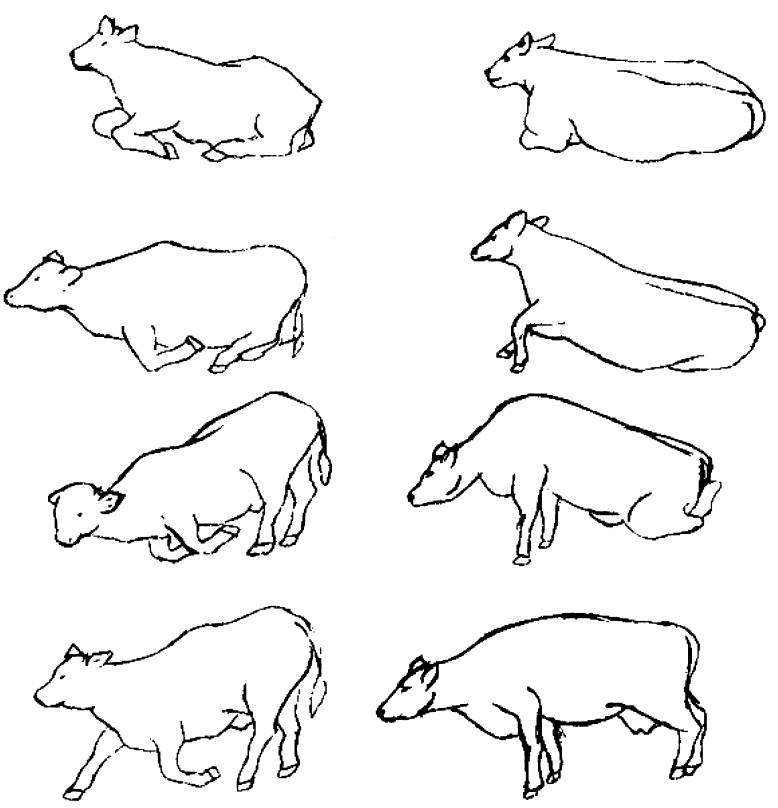

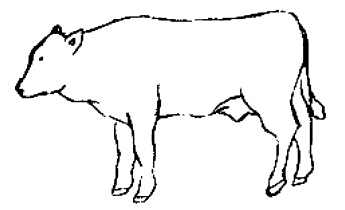

(a)

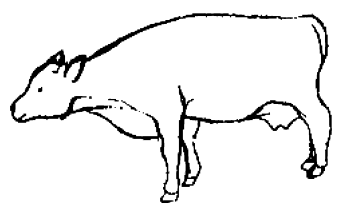

(b)
Figure 1. Getting-up movement of cows: a) right, b) wrong (drawings by Luca Vinci, adapted from Chiappini \& Barbari, 1983 and Fostier et al., 1985). 
With the exception of some numerical measures (stall and feed trough dimensions, average cattle weight), all other answers were coded as binary or discrete variables (Appendix 1) in order to be statistically analysed. Continuous variables were analysed by univariate descriptive statistics (SAS, 1985); non-continuous variables were expressed as absolute and relative frequencies in each of the three villages. On some farms there were more than one building, often with different characteristics. When variables were related to the characteristics of each building, the results are presented for each building $(n=54)$; when they were related to the general characteristics of each farm, they are presented for each farm $(n=47)$.

Principal Component Analysis (PCA) was used in order to highlight the relationships among housing and management traits and cows' welfare. PCA was considered a suitable method to treat this data set, as it presents a number of advantages. First, it is a chemometric statistical method which condenses into few latent variables the information contained in many original variables. Secondly, it offers the possibility of using discrete variables as well as continuous variables, as it is absolutely independent of data distribution; furthermore, the use of PCA with binary variables allows for qualitative considerations (Jolliffe, 1986; Mattiello et al., 1997a).

Cluster analysis (complete linkage method) was performed with the aim of finding groups with homogeneous characteristics of management and welfare. This is also a suitable method for the analysis of non-normally distributed data, which allows allocation of the experimental units to different groups, on the basis of their similarity level and on the possibility of interpreting the results (Kaufman \& Rousseeuw, 1990; Mattiello et al., 1997b). Multivariate analysis was carried out on the 54 buildings, using 24 selected variables (18 non-continuous variables, coded in Appendix 1, and six continuous variables), listed in Table IV.

\section{Results}

Some general and management characteristics are reported in Table I. Most of the farmers are older in Lillianes (mode: 51 years, range $27-77$ years) than in PSM (mode: 39 years, range $23-70$ years) and in Perloz (mode: 43 years, range $34-55$ years). Cattle weight is lower in Lillianes (mode: $500 \mathrm{~kg}$, range $425-530 \mathrm{~kg}$ ) than in PSM (mode: $550 \mathrm{~kg}$, range $450-600 \mathrm{~kg}$ ) and Perloz (mode: $550 \mathrm{~kg}$, range $550-$ $600 \mathrm{~kg}$ ). One stall row is present only in $24 \%$ of buildings in PSM, in $33.3 \%$ in Perloz and 40\% in Lillianes. Seventy-eight per cent of the two row buildings still adopt the back-to-back practice
Table I. General characteristics of farms and management in the three villages.

\begin{tabular}{|c|c|c|c|}
\hline & Lillianes & PSM & Perloz \\
\hline \multicolumn{4}{|l|}{ Age of construction ${ }^{\mathrm{a}}$} \\
\hline Before 1900 & $6(40.0 \%)$ & $5(25.0 \%)$ & $2(28.6 \%)$ \\
\hline $1901-1970$ & $1(6.7 \%)$ & $5(25.0 \%)$ & $0(0.0 \%)$ \\
\hline After 1970 & $8(53.3 \%)$ & $10(50.0 \%)$ & $5(71.4 \%)$ \\
\hline \multicolumn{4}{|l|}{ Number of cattle } \\
\hline$<10$ head & $3(18.8 \%)$ & $4(17.4 \%)$ & $1(12.5 \%)$ \\
\hline $11-30$ head & $7(43.8 \%)$ & $9(39.1 \%)$ & $4(50.0 \%)$ \\
\hline$>30$ head & $6(37.5 \%)$ & $10(43.5 \%)$ & $3(37.5 \%)$ \\
\hline \multicolumn{4}{|c|}{ Duration of indoor housing period } \\
\hline $120-160$ days & $7(43.8 \%)$ & $16(69.6 \%)$ & $1(12.5 \%)$ \\
\hline $161-200$ days & $8(50.0 \%)$ & $4(17.4 \%)$ & $6(75.5 \%)$ \\
\hline $201-240$ days & $1(6.3 \%)$ & $3(13.0 \%)$ & $1(12.5 \%)$ \\
\hline \multicolumn{4}{|c|}{ Duration of spring grazing period } \\
\hline$<30$ days & $12(75.0 \%)$ & $7(30.4 \%)$ & $5(50.0 \%)$ \\
\hline $31-60$ days & $4(25.0 \%)$ & $12(52.2 \%)$ & $3(37.5 \%)$ \\
\hline$>60$ days & $0(0.0 \%)$ & $4(17.4 \%)$ & $1(12.5 \%)$ \\
\hline \multicolumn{4}{|c|}{ Duration of autumn grazing period } \\
\hline$<30$ days & $11(68.8 \%)$ & $12(52.2 \%)$ & $6(75.0 \%)$ \\
\hline $31-60$ days & $5(31.3 \%)$ & $10(43.5 \%)$ & $1(12.5 \%)$ \\
\hline$>60$ days & $0(0.0 \%)$ & $1(4.3 \%)$ & $1(12.5 \%)$ \\
\hline \multicolumn{4}{|l|}{ Milking procedures } \\
\hline Hand milking & $8(50.0 \%)$ & $10(43.5 \%)$ & $3(37.5 \%)$ \\
\hline Mixed procedures ${ }^{\mathrm{b}}$ & $3(18.8 \%)$ & $3(13.0 \%)$ & $2(25.0 \%)$ \\
\hline Mechanical milking & $5(31.3 \%)$ & $10(43.5 \%)$ & $3(37.5 \%)$ \\
\hline \multicolumn{4}{|l|}{ Presence of bull } \\
\hline No & $14(87.5 \%)$ & $16(69.6 \%)$ & $6(75.0 \%)$ \\
\hline Yes & $2(12.5 \%)$ & $7(30.4 \%)$ & $2(25.0 \%)$ \\
\hline
\end{tabular}

${ }^{a}$ Five farmers did not answer.

b Some farmers adopted both milking methods, as they preferred using hand milking for particularly nervous cows.

(28/37), with a central walkway and feed troughs placed by the wall. The largest stall size was recorded in PSM (Table II). Partitions between stalls are never present (except in one building, where cows are in cubicles). Feed trough width is $40-50 \mathrm{~cm}$, while wall height is around $60 \mathrm{~cm}$ (Table III). In most buildings, the feed trough is placed by the wall (75.5\% of buildings in Lillianes, $72 \%$ in PSM and $66.7 \%$ in Perloz). Cows are usually tied by a tether fixed to the feed trough (which severely limits the animal's movements, as the tether junction is fixed), except for one case where there is a vertical chain

Table II. Stall size in the buildings of each of the three villages.

\begin{tabular}{lrrrrrrrrr}
\hline & & \multicolumn{3}{c}{ Stall width cm } & & \multicolumn{3}{c}{ Stall length cm } \\
\cline { 3 - 5 } \cline { 7 - 9 } & $n$ & mode & $\min$ & $\max$ & & mode & $\min$ & $\max$ \\
\hline Lillianes & 20 & 100 & 100 & 120 & & 160 & 145 & 175 \\
PSM & 25 & 100 & 96 & 150 & & 170 & 150 & 203 \\
Perloz & 9 & 100 & 100 & 125 & & 160 & 150 & 175 \\
Overall & 54 & 100 & 96 & 150 & & 160 & 145 & 203 \\
\hline
\end{tabular}


Table III. Characteristics of the feed trough (width, height of the wall by the cow and height of the bottom from stall platform) in the buildings of each of the three villages.

\begin{tabular}{|c|c|c|c|c|c|c|c|c|c|c|}
\hline & \multirow[b]{2}{*}{$n$} & \multicolumn{3}{|c|}{ Feed trough width $(\mathrm{cm})$} & \multicolumn{3}{|c|}{ Height of the wall $(\mathrm{cm})$} & \multicolumn{3}{|c|}{ Height from stall platform $(\mathrm{cm})$} \\
\hline & & mode & $\min$ & $\max$ & mode & $\min$ & $\max$ & mode & $\min$ & $\max$ \\
\hline Lillianes & 20 & 40 & 40 & 62 & 60 & 50 & 70 & 30 & 25 & 40 \\
\hline PSM & 25 & 50 & 30 & 70 & 60 & 20 & 100 & 30 & 0 & 50 \\
\hline Perloz & 9 & 50 & 40 & 65 & 60 & 40 & 70 & 35 & 18 & 45 \\
\hline Overall & 54 & 40 & 30 & 70 & 60 & 20 & 100 & 30 & 0 & 50 \\
\hline
\end{tabular}

tying system (where the tether junction can move up and down along a vertical bar, thus allowing more possibility of movement for the cow), and another case where cows are in cubicles, and their movements are limited by the feed trough rail in the front and by a chain at the back (this was the less restrictive system).

Almost all of the buildings have a concrete floor. The litter is made of straw or sawdust, and its amount is often quite limited $(1.5 \mathrm{~kg} / \mathrm{head} /$ day or less); only in three farms an amount of $2.5 \mathrm{~kg} / \mathrm{head} /$ day is distributed. Water bowls are present in all of the buildings visited in Perloz, but they are absent in $16 \%$ of the buildings in PSM and in $40 \%$ of the buildings in Lillianes. In the 42 buildings in which water bowls are present, water is available ad libitum only in $41.7 \%$ of cases in Lillianes, $47.6 \%$ in PSM and $52.4 \%$ in Perloz. In the remaining cases, water is restricted. In the absence of water inside the building, cows are taken to drink to a common fountain twice a day. Smell perception is better in PSM (44\% neutral, 44\% medium, $12 \%$ nauseating) and Perloz $(66.7 \%$ neutral, $22.2 \%$ medium, $11.1 \%$ nauseating) than in Lillianes ( $0 \%$ neutral, $90 \%$ medium, $10 \%$ nauseating). Wall connections are tight in most of the buildings $(100 \%$ in PSM, $89.9 \%$ in Perloz and $70 \%$ in Lillianes). Health problems due to teat trauma are present in PSM (34.8\% of farms), and nearly absent in the other two villages. Lameness can be considered a problem in $31.9 \%$ of the farms, with no differences among villages. Getting up occurs mostly in a normal way only in four out of 44 farms, while it occurs more frequently with unnatural movements in 38 farms and it is performed in both ways in the remaining two farms. Most farmers were surprised by this question: many of them considered the unnatural movements as part of the cows' normal behavioural repertoire, and three did not answer. One of the farms where both types of getting- up movements were recorded is that in which two tie systems are present in two different buildings: in the building where cows are tethered with a chain to the feed trough, the more common transition movement is abnormal, while it is normal where cows are in cubicles. Tongue playing has been recorded in $18.8 \%$ of the farms in Lillianes, $43.5 \%$ in PSM and $25 \%$ in Perloz. Water lapping is also a common problem in the farms in which water is available to the animals $(33.3 \%$ of the farms in Lillianes, $30 \%$ in PSM and $75 \%$ in Perloz).

The first four principal components (PC) explain $49.2 \%$ of the total variance. Description of PC1 ranges from buildings with higher numbers of animals, more modern facilities (two stall rows, feed trough by the walkway), a higher level of mechanization (for milking and manure removal), better management (higher frequency of manure removal, provision of water), better environmental parameters (ventilation and wall connections) and higher incidence of behavioural problems (tongue playing, water lapping) to old fashioned buildings, which are characterized by the presence of only one stall row, where feed troughs usually have higher walls, with the bottom at a higher level, usually placed by the wall (Figure 2a). The plot of the different farms on the first two PCs (which explain the higher percentage of total variance) shows that most of the buildings with modern characteristics and better management are located in PSM, while old buildings can be found mainly in Lillianes (Figure 2b). On PC2 there is a clear relationship between the duration of spring and autumn grazing periods. On PC3 we find additional information about the direct correlation between water provision and water lapping (Figure 3). On PC4 we can note that lameness is less frequent when manure is frequently removed and that teat trauma is more common in narrower stalls. Cluster analysis allowed identifying four clusters of buildings (Table IV). Cluster 4 is a small homogeneous group of new buildings in PSM, characterized by a high number of animals, generally good management and a high level of mechanization; however, the presence of behaviour problems (especially tongue playing, abnormal getting-up movements and, to a lesser extent, water lapping) and teat trauma is common. Cluster 2 contains most of the very old buildings, characterized by old-fashioned housing systems, with a low level of mechanization and rather bad 


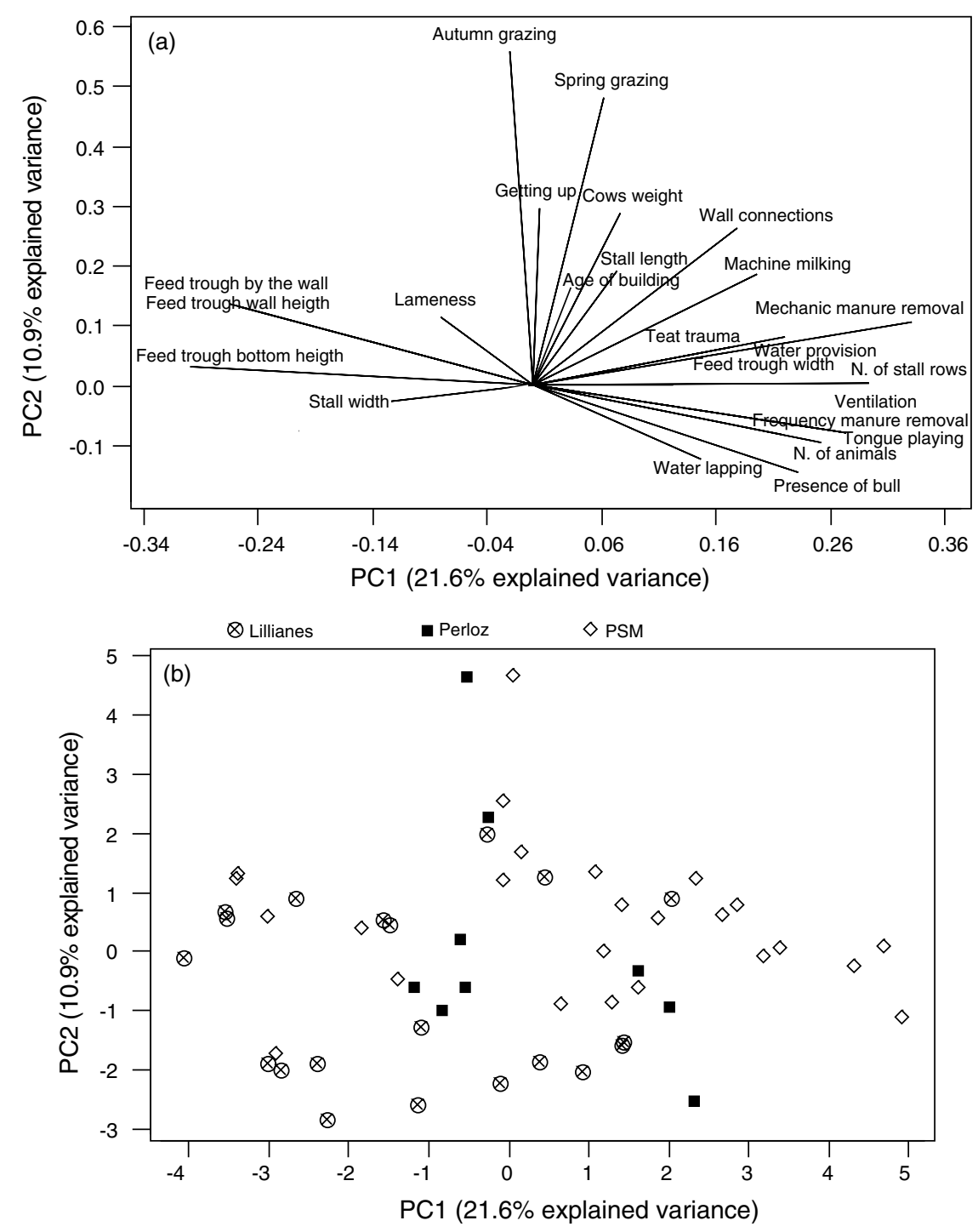

Figure 2. a) Loadings of variables on PC1 and PC2, b) Plot of the buildings, showing how these buildings cluster in relation to PC1 and PC2.

management; in these buildings, behaviours such as tongue playing and water lapping do not represent a problem, although getting-up movements are abnormal, and lameness is a common problem. In clusters 1 and 3 we find intermediate situations from different villages (Table IV).

\section{Discussion}

The interviewer (a veterinary officer from the local health board) routinely visited the surveyed farms; this allowed him to check the reliability of the farmers' answers, which was considered acceptable for all of the farms.

Differences among the three villages are evident and reflect different geographical situations. At the bottom of the valley, where areas are flatter (especially in PSM), the duration of the grazing period is longer. This is obviously related to the availability of larger pasture areas. In contrast, cows are housed in tie-stalls for longer periods in Perloz and Lillianes. Lillianes, which lies on steep slopes and has a surface area smaller than Perloz, presents the most difficult situation. The buildings are usually quite old and the pasture available is limited, so cows are housed for longer periods. Conditions of life are harder, and this is probably the reason why the farmers are usually older. The old age of the structures together with the old age of the farmers are responsible for the less efficient management in Lillianes and, to a lesser extent, in Perloz. Having the higher number of farms, PSM presents the more heterogeneous situation, with some very modern buildings and some of the oldest ones (Table IV). One of the main problems of old buildings is represented by the shape of the feed trough. In these buildings, the 


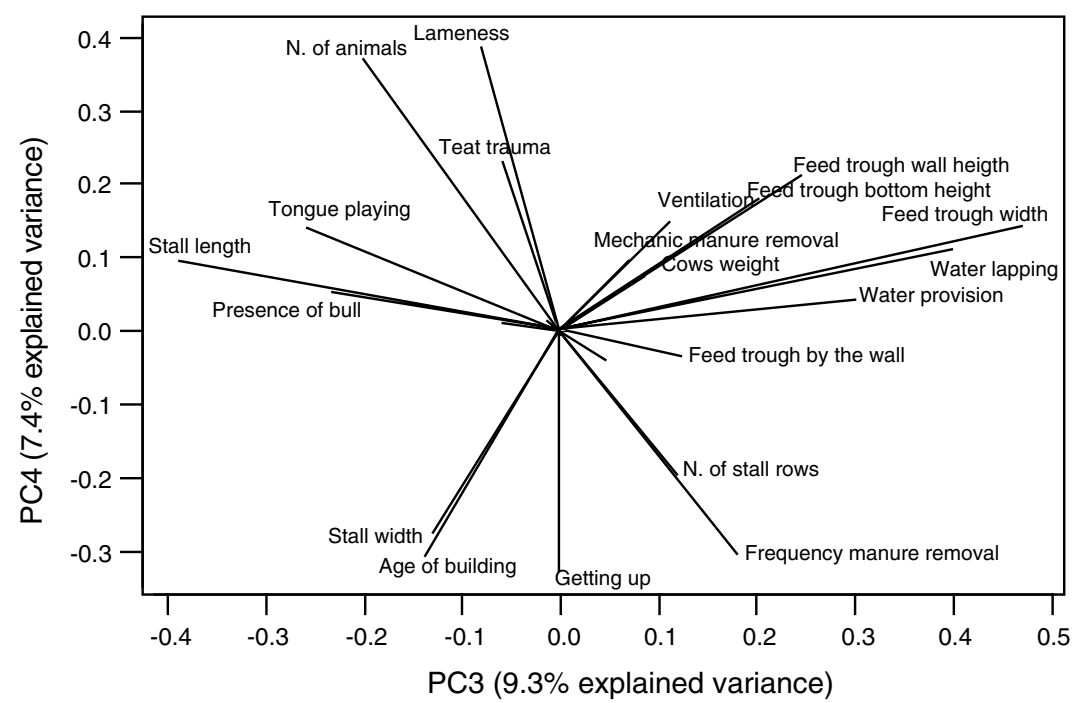

Figure 3. Loadings of variables on PC3 and PC4. Variables with loadings lower than 0.10 on both PCs do not appear in figure, although they were used for the analysis.

feed trough is usually placed by the wall (this is common in the old 'back-to-back' practice), in order to limit feed waste. For the same reason, the trough wall is usually higher than the recommended value of $20 \mathrm{~cm}$ (Bovagne \& Frayer, 1998), thus hampering the cows' getting-up movement. A high level of the feed trough bottom from the stall platform is a further limitation to the waste of forage, but it does not meet the ethological requirements of cattle, which are used to grazing at their feet level (Maton et al., 1985). Stall width is often close to, or even below, the minimum recommended values $(105 \mathrm{~cm})$ (Bovagne \& Frayer, 1998) for guaranteeing satisfactory comfort and hygiene of the cows in mountain housing structures. Furthermore, according to Maton et al. (1985) stall width below $110 \mathrm{~cm}$ may have negative effects on animal welfare, as it induces a decrease of lying time. Stall length for cows

Table IV. Main characteristics of the four clusters identified by cluster analysis.

\begin{tabular}{|c|c|c|c|c|}
\hline ‘ & $\begin{array}{c}\text { Cluster } 1 \\
(n=20: \text { Lillianes }=4, \\
\text { PSM }=9, \text { Perloz }=7)\end{array}$ & $\begin{array}{c}\text { Cluster } 2 \\
(n=17: \text { Lillianes }=13, \\
\text { PSM }=4, \text { Perloz }=0)\end{array}$ & $\begin{array}{c}\text { Cluster } 3 \\
(n=10: \text { Lillianes }=2, \\
\operatorname{PSM}=6, \text { Perloz }=2)\end{array}$ & $\begin{array}{c}\text { Cluster } 4 \\
(n=7: \text { Lillianes }=0, \\
\text { PSM }=7, \text { Perloz }=0)\end{array}$ \\
\hline Age of building & old & very old & new & very new \\
\hline Number of animals & medium & low & low & large \\
\hline Cow weight & high & low & medium/high & medium/low \\
\hline Presence of bull & yes/no & no & no & yes \\
\hline Autumn grazing duration & short & short & long & medium \\
\hline Spring grazing duration & short & short & medium & long \\
\hline Freq. of manure removal & frequent & rare & medium/frequent & frequent \\
\hline Manure removal & mechanical & by hand & mechanical/by hand & mechanical \\
\hline Milking & mechanical/by hand & by hand & mechanical & mechanical \\
\hline Water provision & high & low & high & medium \\
\hline Number of stall rows & two & one & two & two \\
\hline Stall width & medium & large & narrow & very narrow \\
\hline Stall length & short & medium & medium & long \\
\hline Feed trough bottom height & low & high & medium & very low \\
\hline Feed trough wall height & low & medium & high & very low \\
\hline Feed trough width & large & narrow & narrow/medium & narrow/medium \\
\hline Feed trough by the wall & yes/no & yes & yes & no \\
\hline Ventilation & medium/adequate & scarce & medium/scarce & adequate \\
\hline Wall connections & medium/tight & loose & tight & tight \\
\hline Teat trauma & medium & rare & rare & frequent \\
\hline Lameness & frequent & frequent & medium & rare \\
\hline Getting up & abnormal & abnormal & normal & abnormal \\
\hline Water lapping & yes & no & yes/no & yes/no \\
\hline Tongue playing & no/yes & no & yes/no & yes \\
\hline
\end{tabular}


weighing between 500 and $550 \mathrm{~kg}$ should be $160-165 \mathrm{~cm}$ (Bovagne \& Frayer, 1998), which is close to the values recorded in the present research, although some stalls were shorter. Together with other characteristics of the housing structures (as discussed below), short stalls may be responsible for the presence of abnormal getting-up behaviour, and therefore they may cause welfare problems (Chaplin \& Munksgaard, 2001).

Many general management aspects of the visited farms were questionable. The daily amount of litter was below the minimum recommended in order to guarantee sufficient comfort to the animals when they lie down and during transition movements $(2 \mathrm{~kg}$ of straw) (Maton et al., 1985), and cannot guarantee adequate comfort to the animals. Water provision was often limited; according to the farmers, this aimed to control the arousal of water lapping, which causes wet litter. Restricted water provision may have detrimental effects on milk production, as cows are unable to satisfy their water requirements (Herren, 1994). However, water lapping is actually a frequent problem when water is supplied to the cows. Obviously, the absence of this abnormal behaviour in cows deprived of water does not mean that these cows are in better welfare conditions, and the fact that when water is available cows exhibit water lapping can be interpreted as an indication of boredom from suppressed grazing behaviour and lack of exercise (Albright \& Arawe, 1997). Tongue playing was present in one-third of the farms, especially in large farms with large numbers of animals. Personal observations with veal calf facilities suggest the hypothesis that in smaller farms there is a quieter environment which is more favourable to the animals and which does not particularly stimulate the arousal of this stereotypical behaviour. The arousal of abnormal behaviour, such as stereotypies, is considered an indicator of poor welfare or of discomfort situations (Metz \& Wierenga, 1987). Redbo (1990, 1992, 1993) reports that tongue playing is a frequent behaviour problem in tethered cows, especially at the beginning of the tethering period, and suggests that tethering may induce it in dairy cows in response to the deprivation of normal feeding behaviour.

Getting-up behaviour was unnatural in most of the visited farms, regardless of the housing design or management. Problems with getting-up behaviour may result in lesions, and may be therefore associated with poor animal welfare (Chaplin \& Munksgaard, 2001). The high incidence of an abnormal getting-up behaviour is probably due to the tether system, to inadequate housing structures (e.g., short stalls) and/or to the presence of a physical barrier (e.g., feed trough wall) before the animals, which prevents the normal movement. In fact, for getting up in a normal way, cattle need about $70 \mathrm{~cm}$ of head room for a forward lunge; if they do not have enough space or if they meet some physical barrier before them, they get up by raising the front part of the body and subsequently the hind one, which is the opposite of what they would normally do (Rist \& Scharagel, 1996; Albright \& Arawe, 1997).

This unnatural behaviour is so widespread that the farmers consider it normal. This raises some considerations: first, there is some lack in the farmers' knowledge of cattle behaviour; secondly, in the considered housing system cows are not allowed to exhibit their normal behaviour, possibly in relation to several factors, such as short stalls, front barriers and/or the use of tethers. The use of tethering systems alternative to the chain, such as cows in cubicles with back chains, as described above, seems to be a possibility for allowing the cows to maintain a more natural behaviour, although this is simply a hypothesis arising from a single observation. Modern farms have a higher level of mechanization and good management. However, higher investment must be presumably followed by higher profitability of the enterprises: this means that modern farms often try to intensify production by housing more animals per surface area. This can be achieved, for example, by reducing stall width. This is probably one of the reasons why modern farms (cluster 4 ) have a higher presence of teat trauma than very traditional farms, where the larger stall dimension probably affects the lesser incidence of teat trauma (cluster 2). A negative correlation between stall width and frequency of teat trauma is shown also on PC1. Partitions of $80-100 \mathrm{~cm}$ between animals may reduce the incidence of teat trauma (Chiappini \& Barbari, 1983); their lack probably contributes to the occurrence of this health problem. The inverse relationship between age of the building and presence of teat trauma suggests that modern farms are not necessarily better than traditional farms in guaranteeing high welfare levels, although this happens in some cases. Modern farms usually have a higher level of mechanization, which facilitates cleaning operations. This seems to have positive effects on the health of the animals. In clusters 2, 3 and 4, the incidence of lameness appears to be inversely associated with the frequency of manure removal (see also PCA, Figure 3). However, in cluster 1, lameness can be considered a problem, in spite of the presence of frequent mechanical manure removal; in this case, the short time spent at pasture probably also plays a role in the occurrence of lameness. This is supported by the fact that Singh et al. (1992) found a higher incidence of sole lesions in housed cows than in cows 
on pasture. A long duration of grazing periods, associated with frequent manure removal during the housing period, are probably key factors for limiting the occurrence of podal lesions.

The present study represents an attempt to draw attention to the welfare problems of cattle in tiestalls. Many welfare problems may also be present in loose-housing systems; however, tie-stalls are a more limiting system in terms of possibility of movement and of social contacts, and may cause more serious welfare problems. On the whole, the welfare status of the surveyed herds seems to be low. Some aspects of this complex problem are taken into account in the present survey. Obviously, several other factors can affect the well-being of tethered cows. Although many welfare problems may occur in this housing system, tie-stalls are the only system which is presently feasible in mountain areas, where it is difficult to have enough space for loose housing, where the animals must be kept indoors during the winter and where it is difficult to take the animals to pasture areas for long periods. This is the case for Valle d'Aosta, which is characterized by high altitudes and steep slopes. Therefore, even though it seems difficult to guarantee high welfare levels in tethered cows, it is important to understand which critical points can be improved. In response to different geographical characteristics, remarkable differences are present even among three neighbouring villages located in the same Italian region. This suggests that some characteristics are strictly related to some physical constraints (e.g., land layout and conformation), and therefore some changes are difficult to achieve. However, circumstances permitting, it seems that some expedients may be practised. For example, it may be useful to maintain the animals at pasture as long as possible, and this is more easily achievable in a village such as PSM, which is located at a lower altitude and in a flatter area; frequent manure removal and the provision of water can also be suggested, and this can be done in any geographical situation, although they demand an increase in labour; to design stalls with dimensions adequate for cows' size and which leave enough front space for allowing the animals to perform their normal getting-up behaviour is a further improvement which can be suggested and which can be carried out in any geographical situation, but it is obviously an expensive intervention. The answers from the farmers highlighted some lack in their knowledge of animal behaviour. We believe that the knowledge of animal behaviour is important in order to guarantee good welfare levels. Specific training courses for the stockpersons may help to provide an additional improvement in animal welfare.

\section{Acknowledgements}

We are grateful to all the farmers who answered to our questionnaires and to Professor Roberto Todeschini for his assistance with statistical analysis.

\section{References}

Alban, L. (1995). Lameness in Danish dairy cows: frequency and possible risk factors. Preventive Veterinary Medicine, 22, 213 225 .

Albright, J., \& Arawe, C. (1997). The behaviour of cattle. Cab International, Oxon. 320 pp.

Bovagne, J., \& Frayer, C. (1998). Guide pour la conception et l'amenagement des bâtiments d'élevage bovin dans les regions de montagne, Project Interregional n. 105. Aosta, Chambre d'Agriculture de la Haute Savoie, Assessorat de l'Agriculture et des Ressources Naturelles, Region Autonome Vallée d'Aoste. 90 pp.

Bloom, J. (1983). Traumatic injuries and foot diseases related to housing systems. Farm animals and welfare. Martinus Nijhoff Publishers, Boston, MA. 58 pp.

Centro Ricerche Produzioni Animali . (1999). Stalle per vacche da latte, 2nd edn, Edizioni L'Informatore Agrario SpA, Verona, Italy. $63 \mathrm{pp}$.

Charron, G. (1998). Les productions laitières, volume 2. J.B. Baillière, Paris, France. 128 pp.

Chaplin, S., \& Munksgaard, L. (2001). Evaluation of a simple method for assessment of rising behaviour in tethered dairy cows. Animal Science, 72, 191-197.

Chiappini, U., \& Barbari, M. (1983). Stalle per vacche da latte. In: U. Chiappini (Ed.), Ricoveri zootecnici e attrezzature . Reda, Roma, Italy. pp 54-77.

Cook, N.B. (2004). Sand stalls, sore feet, and sour rumens North American perspectives on lameness in dairy cows. Cattle Practice, 12, 275-280.

Fostier, B., Soissons, J., \& Tillie, M. (1985). Pathologie et logement des bovins. Iteb, Paris, France. 154 pp.

Jolliffe, I.T. (1986). Principal Component Analysis. SpringerVerlag, New York, NY, 502 pp.

Kaufman, L., \& Rousseeuw, P.J. (1990). Finding groups in data. An introduction to cluster analysis. John Wiley \& Sons Inc., New York, NY, 342 pp.

Hagen, K., Lexer, D., Palme, R., Troxler, J., \& Waiblinger, S. (2004). Milking of Brown Swiss and Austrian Simmental cows in a herringbone parlour or an automatic milking unit. Applied Animal Behavioural Science, 88, 209-225.

Herren, V.R. (1994). The science of animal agriculture. Delmar Publishers, New York, NY, 335 pp.

Main, D.C.J., Webster, A.J.F., \& Green, L.E. (2001). Animal welfare assessment in farm assurance schemes. Acta Agriculturae Scandinavica, Section A, Animal Science, Supplement, 30, $108-113$.

Maton, A., Daelemans, J., \& Lambrecht, J. (1985). Housing of animals. Elsevier, Amsterdam, The Netherlands, 458 pp.

Mattiello, S., Todeschini, R., \& Verga, M. (1997a). Applicazioni chemiometriche nel campo delle scienze zootecniche. Nota I: esplorazione dei dati ed analisi di regressione. Zoot. Nutr. Anim., 23, 105-116.

Mattiello, S., Verga, M., \& Todeschini, R. (1997b). Applicazioni chemiometriche nel campo delle scienze zootecniche. Nota II: cluster analysis e classificazione. Zoot. Nutr. Anim., 23, $167-178$.

Metz, J., \& Wierenga, H. (1997). Behavioural criteria for the design of housing systems for cattle. Cattle housing systems, 
lameness and behaviour. Martinus Nijhoff Publishers: Boston, MA. 112 pp.

Rist, H., \& Schragel, I. (1996). Allevamento etologico dei bovini. Edagricole, Bologna, Italy. 228 pp.

Redbo, I. (1990). Changes in duration and frequency of stereotypes and their associated behaviours in heifers before, during and after the grazing period. Applied Animal Behavioural Science, 26, 57-67.

Redbo, I. (1992). The influence of restraint on the occurrence of oral stereotypes in dairy cows. Applied Animal Behavioural Science, 35, 115-123.

Redbo, I. (1993). Stereotypes and cortisol secretion in heifers subjected to tethering. Applied Animal Behavioural Science, $38,213-225$.

Regula, G., Danuser, J., Spycher, B., \& Wechsler, B. (2004). Health and welfare of dairy cows in different husbandry systems in Switzerland. Preventive Veterinary Medicine, 66, $247-264$.

Rousing, T. (2003). Welfare assessment in dairy cattle herds with loose-housing cubicle systems. Development and evaluation of welfare indicators. Ph.D. Thesis, Danish Institute of Agricultural Sciences, Department of Animal Health and Welfare \& Royal Veterinary and Agricultural University, Department of Animal Science and Animal Health. DIAS report, Animal Husbandry 45. 101 pp.

SAS. (1985). SAS User's Guide: Statistics, Version 5. SAS Inst. Inc., Cary, NC. 956 pp.
Singh, S.S., Murray, R.D., \& Ward, W.R. (1992). Histopathological and morphometric studies on the hooves of dairy and beef cattle in relation to overgrown sole and laminitis. $\mathcal{F}$. Comp. Pathol., 107, 319-328.

Sørensen, J.T., Sandøe, P., \& Halberg, N. (2001). Animal welfare as one among several values to be considered at farm level: the idea of an ethical account for livestock farming. Acta Agriculturae Scandinavica Section A, Animal Science, Supplement, 30, 11-16.

Valde, J.P., Hird, D.W., Thurmond, M.C., \& Osteras, O. (1997). Comparison of ketosis, clinical mastitis, somatic cell count, and reproductive performance between free-stall and tiestall barns in Norwegian dairy herds with automatic feeding. Acta Veterinarae Scandinavica, 38, 181-192.

Waiblinger, S., Menke, C., \& Folsch, D.W. (2003). Influences on the avoidance and approach behaviour of dairy cows towards humans on 35 farms. Applied Animal Behavioural Science, 84, 23-39.

Wiepkema, P.R., Broom, D.M., Duncan, I.J.H., \& van Putten, G. (1983). Abnormal behaviours in farm animals. A Report of the Commission of the European Communities: Luxembourg. $44 \mathrm{pp}$.

Winckler, C., Capdeville, J., Gebresenbet, G., Horning, B., Roiha, U., Tosi, M.V., \& Waiblinger, S. (2003). Selection of parameters for on-farm welfare-assessment protocols in cattle and buffalo. Animal Welfare, 12, 619-624.

\section{Appendix 1. Variables codes}

Age of building $\left(\mathrm{QF}^{1}\right)$ : before $1900=1 ; 1901-1970=2$; after $1970=3$.

Number of animals $(\mathrm{QF})$ : less than 10 cows $=1 ; 11-30$ cows $=2$; more than 30 cows $=3$.

Presence of bull $\left(\mathrm{DR}^{2}\right)$ : no $=1$; yes $=2$.

Spring grazing duration $(\mathrm{QF})$ : less than $30 \mathrm{~d}=1 ; 30-60 \mathrm{~d}=2$; more than $60 \mathrm{~d}=3$.

Autumn grazing duration $(\mathrm{QF})$ : less than $30 \mathrm{~d}=1$; 30-60 d=2; more than $60 \mathrm{~d}=3$.

Frequency of manure removal $(\mathrm{QF})$ : once/d $=1$; twice $/ \mathrm{d}=2$.

Manure removal $(\mathrm{QF})$ : manual $=1$; mechanic (dung scraper) $=2$.

Milking $(\mathrm{QF})$ : manual $=1$; mixed (mechanic and manual $)=2$; mechanic $=3$.

Water provision in the stall $(\mathrm{QF})$ : no water $=0$; restricted water $=1$; free water $=2$.

Number of stall rows (DR): one $=1$; two $=2$.

Feed trough by the wall (DR): no $=1$; yes $=2$.

Ventilation $(\mathrm{DR})$ : scarce $=0$; medium $=1$; adequate $=2$.

Wall connections (DR): loose (fissured walls, with possibility of draughts coming through the cracks) $=1$;

tight (good connections, no draughts) $=2$.

Teat trauma $(\mathrm{QF}):$ no $=1$; yes $=2$.

Lameness $(\mathrm{QF}):$ no $=1$; yes $=2$.

Getting up $(\mathrm{QF})$ : abnormal $=1$; normal $=2$.

Water lapping $(\mathrm{QF}): \mathrm{no}=1$; yes $=2$.

Tongue playing $(\mathrm{QF}): \mathrm{no}=1$; yes $=2$.

${ }^{1} \mathrm{QF}=$ Question to the farmer.

${ }^{2} \mathrm{DR}=$ Direct remark. 